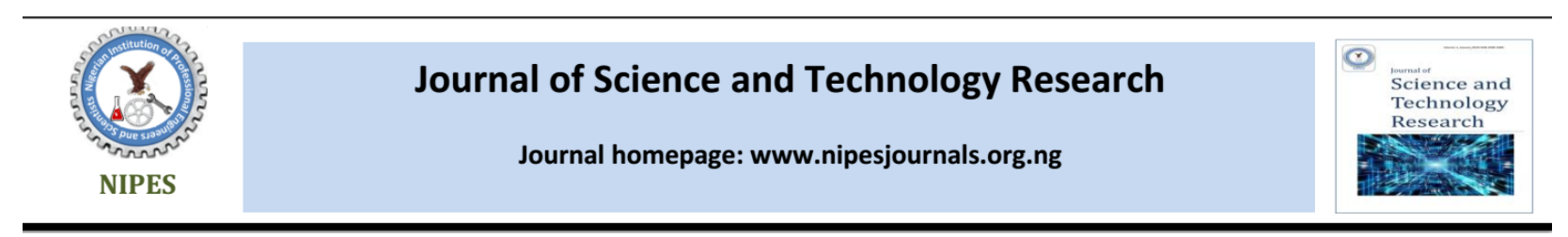

\title{
The Analytical Modeling of Nuclear Size Effect on Relativistic Electron in Hydrogen Atom
}

\author{
Aliyu Adamu ${ }^{a^{*}}$, Adamu Hassan ${ }^{b}$, Ahmad Abdulkadir $^{a}$ and Adamu Z. Ngaric \\ ${ }^{a}$ Department of Physics, Faculty of Science, University of Maiduguri, P. M. B. 1069, Maiduguri - Nigeria \\ ${ }^{b}$ Muhammed Goni College of Legal \& Islamic Studies, P.M.B. 1526, Maiduguri - Nigeria \\ ${ }^{c}$ Department of Physics, Nigerian Army University Biu - Nigeria \\ *Corresponding Author: aliyuadamu703@gmail.com
}

\begin{tabular}{|c|c|}
\hline Article Info & Abstract \\
\hline $\begin{array}{l}\text { Article history: } \\
\text { Received 27April } 2020 \\
\text { Revised 19 June } 2020 \\
\text { Accepted } 22 \text { June } 2020 \\
\text { Available online } 31 \text { August } 2020\end{array}$ & $\begin{array}{l}\text { The effects of relativistic motion, the spin-orbit interaction and } \\
\text { Zitterbewegung of an electron, which are of the same order of } \\
\text { magnitude, defined the fine structure correction to hydrogen spectra. } \\
\text { In this work, perturbation theory, as an approximation method is } \\
\text { applied to examine the effects of finite sized of atomic nucleus on } \\
\text { relativistic motion of electron in hydrogen atom. The nuclear finite }\end{array}$ \\
\hline $\begin{array}{l}\text { Keywords: relativistic motion } \\
\text { fine structure } \\
\text { electron } \\
\text { finite-size nucleus } \\
\text { perturbation } \\
\text { energy states }\end{array}$ & $\begin{array}{l}\text { corrections to } 1 \mathrm{~s}, 2 \mathrm{~s}, 3 \mathrm{~s}, 4 \mathrm{~s} \text { and } 5 \mathrm{~s} \text { energy states in hydrogen atom due } \\
\text { to the finite size of nucleus were computed and the results showed that } \\
\text { the nuclear size effects, which is of order of } 10^{-6} \mathrm{eV} \text {, depends on the } \\
\text { size, } A(N, Z) \text { of the nucleus and energy states, } n \text { of relativistic electron. } \\
\text { This suggested that the nuclear size is more effective on relativistic } \\
\text { electron in the lower energy levels of heavy nuclei, as the effect varied } \\
\text { directly with the nuclear size and inversely with the electron states, } n \text {. }\end{array}$ \\
\hline $\begin{array}{c}\text { Crossref } \\
\text { https://doi.org/10.37933/nipes/2.3.2020.5 }\end{array}$ & $\begin{array}{l}\text { motion of an electron. Moreover, a simple model was developed to } \\
\text { predict the energy level variation as a function of the size of the } \\
\text { nucleus. Therefore, this study justifies the effects of nuclear size on } \\
\text { relativistic electron and the measured values are of greatest interest } \\
\text { since it will reveal significant changes of the nuclear structure and } \\
\text { may also improve the knowledge of fine structure correction. }\end{array}$ \\
\hline $\begin{array}{l}\text { https://nipesjournals.org.ng } \\
\text { (C) } 2020 \text { NIPES Pub. All rights } \\
\text { reserved }\end{array}$ & \\
\hline
\end{tabular}

\section{Introduction}

The hydrogen atom is the fundamental two-body system and perhaps the most important tool of atomic physics and the challenge is to calculate its properties to the highest accuracy possible. The study of the hydrogen atom has been at the heart of the development of modern physics [1]. The hydrogen atom has been the testing ground for theoretical atomic physics for over a hundred years. The original quantum mechanics was motivated to model the hydrogen atom and explain its spectrum. In 1912, Niels Bohr proposed the first electronic hydrogen atom model which successfully predicted the main energy levels of the hydrogen atom in the framework of a semiclassical theory based on Planck's hypothesis. But the main spectral lines of hydrogen atom can be described by the Schrödinger equation, without using any postulates [2].

It is well known that, when the lone orbiting electron solely subject to the electrostatic influence of the nucleus, the complete solution, $\widehat{H}_{0}\left|\psi_{n}\right\rangle=E_{n}\left|\psi_{n}\right\rangle$, of eigenfunctions $\psi_{\mathrm{n}}$ and eigenvalues $E_{\mathrm{n}}$ of the Hamiltonian, $\widehat{H}_{0}$, give rise to the allowed energies of an electron, which depends exclusively on 
the principal quantum number, $n$ as $E_{n}=-13.6 \mathrm{eV}(Z / n)^{2}[3-8]$. It is clearly observed that these allowed energies of an electrondo not give an exact description of the interaction between the fermions and the nucleus. Schrödinger equation was derived based on the assumptions that the atomic nucleus has a point-like charge. The simple point-charge nucleus approximation resulting in a well known $Z / r$ potential (Bohric and Schrodinger approximation) is no longer completely adequate as nuclear system takes into account in particular the following interactions, ordered according to their importance: The spin of the electron; Spin-orbit interaction; Relativistic motion of the electron; Hyperfine Structure Correction; Vacuum fluctuations of the electromagnetic fields; Vacuum polarization. These interactions which are usually incorporated via perturbation theory and quantum electrodynamics, lead to the energy splitting such as fine structure splitting, hyperfine structure splitting and the lamb shift. These splitting have been calculated in many literatures see for example [9-18]. The fine structure of hydrogen atom is interpreted as the effects of relativistic motion, the spin-orbit interaction and Zitterbewegung of an electron [19]. These effects, which are always treated together, are of the same order of magnitude when calculated based on point-charge nuclear assumption.

The dependence of the corrections to discrete eigenvalues of the $Z / r$ potential inside the nucleus necessitates a choice of a model for the nuclear potential that modifies its unphysical infinity at the origin [20-21]. The recent electron scattering experiments at Stanford University have established the finite size of the nucleus and Hofstadter and McAllister were first observed this effect $[22,23]$. High-energy electron-scattering measurements have demonstrated clearly the existence of deviations from point-nucleon scattering laws [24,25] which attributed entirely to finite structure effects in the nucleons. The nucleus has a finite size charge distribution due to the fact that its nucleons consist of the combination of $u$-quarks and $d$-quarks of charges $2 e / 3$ and $e / 3$ respectively. The quarks distributions inside nucleons make the charge distribution of nucleus to be finite over a range $R$ (Figure 1).

The early theorist uses analytical descriptions of charge distribution and potentials, which enabled series expansions of analytical solutions of the wavefunctions within and close to the nucleus and for finite nuclear distributions, the possible range exceeds much further. If the nucleus is being described as a finite-size source with a uniform distribution of charges of radius $R$, then the relativistic electron wave function can penetrate to $r \leq R$, and thus the electron spends part of its time inside the nuclear charge distribution, there it feels a very different interaction. This idea is considered in this paper to refine the relativistic motion of an electron in finite size charge assumption since it is under the influence of nuclear charge. The time independent perturbation theory, as an approximation method will be applied to examine the effects of finite-size nuclear on relativistic electron of hydrogen atom and compared to the previously calculated values.

\section{Methodology}

\subsection{Nuclear Potential and Wavefunction}

One of the simplest models of nucleus is the spherically symmetric charge distribution with the corresponding charge density.

$$
\rho(r)=\frac{3 Z e}{4 \pi R^{3}}
$$

where $Z$ is the nuclear charge, $R=r_{0} A^{1 / 3}$, is the effective radius of nucleus. The experimental data indicates that $r_{0} \approx 1.2 \mathrm{fm}[20,21]$. This simple distribution gives a reasonable approximation for the homogeneous distribution and the correct analytical behavior of the electronic wavefunctions at $r=$ 0 and has been used in many early analyses. The effect of the nuclear distribution on atomic properties is proportional to the expectation values of the nuclear distribution. 


$$
\left\langle r^{2}\right\rangle=\frac{3}{5} R^{2}
$$

Thus, the nuclear charge $R$ is effective radius of nucleus, connected with root-mean-square radius as

$$
R=\sqrt{\frac{5}{3}}\left\langle r^{2}\right\rangle^{1 / 2}
$$

The root-mean-square nuclear matter radii $\left\langle r^{2}\right\rangle^{1 / 2}$ and the density distributions contain an important insight on nuclear potentials and nuclear wavefunctions [26-31]. However, several atomic properties depend directly on the wave function close to the nucleus. This gives a reasonable approximation for the homogeneous distribution.

The simple distribution (1) gives the correct analytical behavior of the electronic wavefunctions at $r=0$ and has been used in many early analyses. These expansions are also useful for a general understanding of the effects involved [32]. For spherically symmetric charge distribution $\rho(r)$ inside the nucleus, the interaction between fermions and nucleus can best be described by the leptonnuclear potential energy $U(r)$ :

$$
U(r)=-k e\left[\frac{4 \pi}{r} \int_{0}^{R} \rho\left(r^{\prime}\right) r^{\prime 2} d r^{\prime}+4 \pi \int_{R}^{\infty} \frac{1}{r^{\prime}} \rho\left(r^{\prime}\right){r^{\prime}}^{2} d r^{\prime}\right]
$$

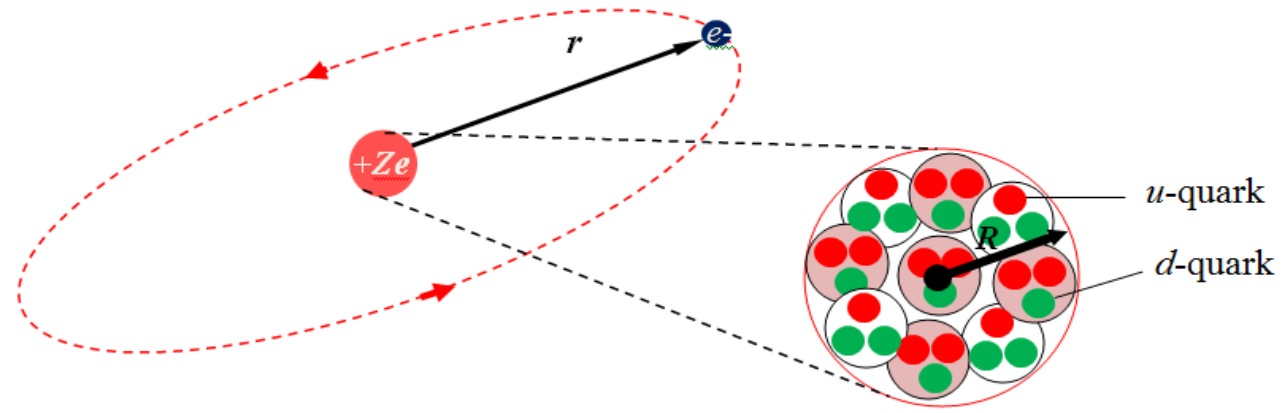

Figure 1: The finite size of atomic nucleus

where outside the nucleus, $r>R$, this expression reduces to a point charge potential

$$
U(r)=-\frac{Z k e^{2}}{r}
$$

and within a nuclear radius $r \leq R$, the expression is described by

$$
U(r, R)=-\frac{Z k e^{2}}{r}\left(\frac{3 r}{2 R}-\frac{r^{3}}{2 R^{3}}\right)
$$

Thus, inside the nucleus of radius $R$, the lepton-nuclearpotential will not have Coulomb form. The constant value of the potential $(5)$ at $(r \sim 0)$ very close to the origin, makes the $Z / r$ potential less singular at small distances and extend, the possible range, much further. The magnitude of the nuclear size effects on hydrogen spectra were accurately calculated by the use of first order time independent perturbation theory:

$$
\widehat{E}_{n}^{(1)}=\lambda\left\langle\psi_{n}^{(0)}\left|\widehat{H}^{\prime}\right| \psi_{n}^{(0)}\right\rangle
$$


which is the superposition of all unperturbed states, $\psi_{n}^{(0)}$.

The first order calculation of the effect of finite size of nucleus with potential (5) gives the energy shift [33]:

$$
\lambda E_{n}=\int \psi_{n}^{(0) *}[U(r, R)-U(r)] \psi_{n}^{(0)} d \tau=\frac{2 Z^{4} k e^{2} R^{2}}{5 n^{3} a_{0}^{3}}=-E_{n}\left(\frac{4 Z^{2} R^{2}}{5 n a_{0}^{2}}\right)
$$

where the use of $d \tau=4 \pi r^{2} d r$ and

$$
E_{n}=-\frac{Z^{2} k e^{2}}{2 n^{2} a_{0}}
$$

The Bohr radius $a_{0}=5.29 \times 10^{-11} \mathrm{~m}$.

\subsection{Relativistic Motion of the Electron of Finite-Size Nucleus}

In relativistic classical mechanics, the total energy of an electron is

$$
E=\sqrt{p_{\mu} p^{\mu}}=m c^{2}+\frac{\vec{p}^{2}}{2 m} \rightarrow \sqrt{(p c)^{2}+\left(m c^{2}\right)^{2}}
$$

In the case where the particle is only slightly relativistic $(v \ll c)$ the square root can be expanded to obtain

$$
E=m c^{2} \sqrt{1+\left(\frac{\vec{p}}{m c}\right)^{2}}=m c^{2}\left[1+\frac{1}{2}\left(\frac{\vec{p}}{m c}\right)^{2}-\frac{1}{8}\left(\frac{\vec{p}}{m c}\right)^{4}+\cdots\right]
$$

The first term in (8) is interpreted as the rest energy of the electron; the second term is the nonrelativistic kinetic energy. Hence, the third term is used in writing the perturbed Hamiltonian:

$$
\lambda \widehat{H}^{\prime}=-\frac{1}{2 m c^{2}}\left(\frac{\vec{p}^{2}}{2 m}\right)^{2}
$$

The new Hamiltonian of this system in terms of finite-size charge distribution takes the form:

$$
\widehat{H}=\frac{\vec{p}^{2}}{2 m}-\frac{Z k e^{2}}{r}\left(\frac{3 r}{2 R}-\frac{r^{3}}{2 R^{3}}\right)
$$

And the perturbed Hamiltonian is given by

$$
\lambda \widehat{H}^{\prime}=-\frac{1}{2 m c^{2}}\left(\frac{\vec{p}^{2}}{2 m}\right)^{2}=-\frac{1}{2 m c^{2}}\left[\widehat{H}^{\prime}+\frac{Z k e^{2}}{R}\left(\frac{3}{2}-\frac{1}{2} \frac{r^{2}}{R^{2}}\right)\right]^{2}
$$

Thus, the relativistic correction for a new state $\left|\psi_{n}\right\rangle$ of relativistic electron can be expressed as:

$$
\begin{aligned}
\Delta E_{r}= & -\frac{1}{2 m c^{2}}\left\langle\psi_{n}\left|\left[\widehat{H}^{\prime}+\frac{Z k e^{2}}{R}\left(\frac{3}{2}-\frac{1}{2} \frac{r^{2}}{R^{2}}\right)\right]^{2}\right| \psi_{n}\right\rangle \approx-\frac{9\left(\lambda E_{n}\right)^{2}}{2 m c^{2}}=-\frac{72 E_{n}^{2}}{25 m a_{0}^{4} c^{2}}\left(\frac{Z^{4} R^{4}}{n^{2}}\right) \\
& =-\left(\frac{r_{0}}{a_{0}}\right)^{4} \frac{72}{25 m c^{2}} \frac{Z^{4} E_{n}^{2} A^{4 / 3}}{n^{2}}
\end{aligned}
$$

where the use of (6) and (7) have been made, $m=9.1 \times 10^{-31} \mathrm{~kg}$ is the electron mass and $c=3.0 \times$ $10^{8} \mathrm{~ms}^{-1}$ is the speed of light in vacuum. The allowed energies of an electron (7) obtained by solving 
Schrödinger equation and the calculated values of finite size nuclear effects to energies (11) obtained from this work are computed for the ground state and first four excited states of relativistic electron in hydrogen atom. Denoting

$$
\zeta=\frac{\Delta E_{r}}{E_{n}}
$$

as the deviation of energy $\Delta E_{r}$ due to finite size of nucleus relative to the energy, $E_{n}$.

\section{Results and Discussion}

Equation (11) gives first order perturbation corrections to energy states of relativistic electron under the influence of finite-size nucleus. The nuclear finite corrections to $1 s, 2 s, 3 s, 4 s$ and $5 s$ energy states in hydrogen atom due to the finite size of nucleus were computed using the results obtained by Equation (11) and the results obtained are presented in Table 1.

Table 1 showed that the nuclear structure effect on relativistic electron, which is of order of $10^{-6} \mathrm{eV}$, depends on the nuclear size, $A(N, Z)$ and the energy states, $n$ of relativistic electron. This suggested that the nuclear size is more effective on relativistic electron in the lower $s$ energy levels of heavy nuclei, as the effect varied directly with the nuclear size and inversely with the quantum number, $n$.

Table 1: The calculated values of finite size nuclear effects to energies of the ground state and first four excited states of relativistic electron in hydrogen atom

\begin{tabular}{cccc}
\hline State & $\boldsymbol{E}_{\boldsymbol{n}}(\boldsymbol{e} \boldsymbol{V})$ & $\Delta \boldsymbol{E}_{\boldsymbol{r}}\left(\mathbf{1 0}^{-\mathbf{6}} \boldsymbol{e} \boldsymbol{V}\right)$ & $\boldsymbol{\zeta} \times \mathbf{1 0}^{-\mathbf{6}}$ \\
\hline $1 s$ & -13.600 & -1703.5 & 125.25 \\
$2 s$ & -3.4000 & -26.617 & 7.8285 \\
$3 s$ & -1.5111 & -2.3367 & 1.5464 \\
$4 s$ & -0.8500 & -0.4158 & 0.4893 \\
$5 s$ & -0.5440 & -0.1090 & 0.2004 \\
\hline
\end{tabular}

The information represented in Table 1 is extended further by plotting a graph of $\log \left(\Delta E_{r}\right)$ as a function of principal quantum number, $n$ (Figure 2).

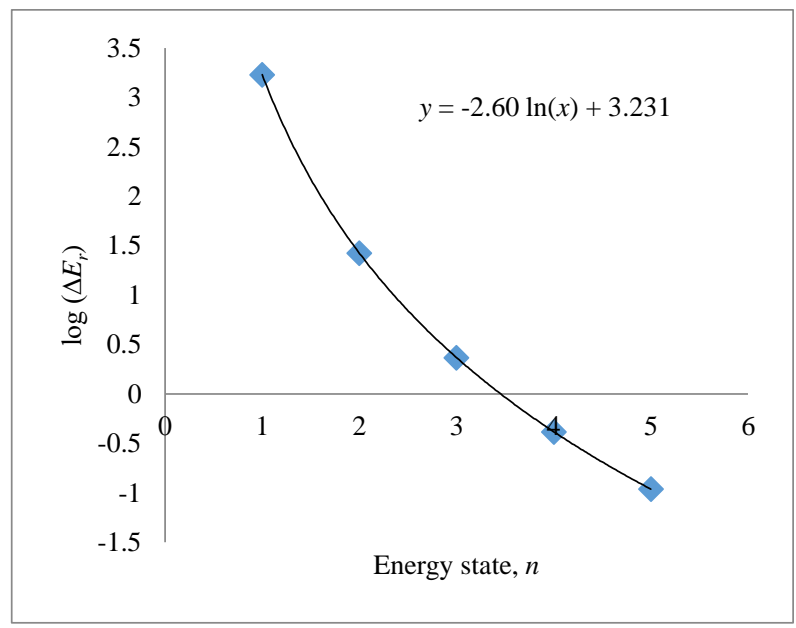

Figure 2: The variation of the magnitude of nuclear finite-size effects, $\log \left(\Delta \boldsymbol{E}_{\boldsymbol{r}}\right)$ on relativistic electron with principal quantum number, $n$.

Figure 2 represents the variation of the magnitude of nuclear finite-size effects, $\log \left(\Delta \boldsymbol{E}_{\boldsymbol{r}}\right)$ on relativistic electron calculated in Table 1 , with principal quantum number, $n$. The figure showed that 
the relativistic effect due to finite size of nucleus decreases with the principal quantum number, $n$. Thus, the nuclear size effect depends on the energy states, $n$ of relativistic electron. This suggested that the nuclear size is more effective on relativistic electron in the lower energy levels of heavy nuclei.Figure 2 showed a very clear continuous curve in decreasing order which points out to be non-linear from the plot of relativistic effect of finite size of nucleus $\log \left[\Delta \boldsymbol{E}_{\boldsymbol{r}}(n, j)\right]$ as a function of the principal quantum number, $n$ for hydrogen atom that corresponds to those recorded in Table 1 by the use of Equation (11). This data can be modeled and studied using the regression analysis. By applying the regression analysis, a simple model satisfying the relationship in Equation (13):

$$
\log \Delta E_{r}(n, j)=-2.60 \ln (n)+3.231
$$

was developed and can predicts the nuclear size effect on relativistic electron. The simple model was used and predicts the energy level variation (up to $n=100$ ) as a function of the size of the nucleus in Table 3 (Appendix). The energy level shifts (11) calculated due to the effect of nuclear size on relativistic electron is compared with the relativistic effect of an electron obtained from Ref. [34] based on the point-charge nucleus calculation. As can be seen from Table 2 that the finite-size nuclear corrections do affects the relativistic electron in any states with values 10 times larger than the relativistic corrections to energy levels of point-charge nucleus.

Table 2: The calculated values of finite size nuclear effects to energies, $\Delta \boldsymbol{E}_{\boldsymbol{r}}$ in the ground state and first four excited states of hydrogen atom obtained from the present work with from point-like nucleus, $\Delta \boldsymbol{E}_{\boldsymbol{n}}$ in $1 s, 2 s, 3 s, 4 s$ and $5 s$ states obtained from Ref. [34].

\begin{tabular}{ccc}
\hline Orbitals & $\Delta \boldsymbol{E}_{n}(\mathbf{e V})$ & $\Delta \boldsymbol{E}_{\boldsymbol{r}} \times \mathbf{1 0}^{-\mathbf{6}}(\mathrm{eV})$ \\
\hline $1 S_{1 / 2}$ & $1.8115 \times 10^{-4}$ & $1.7035 \times 10^{-3}$ \\
$2 S_{1 / 2}$ & $2.8305 \times 10^{-6}$ & $2.6617 \times 10^{-5}$ \\
$2 P_{1 / 2}$ & $2.8305 \times 10^{-6}$ & - \\
$2 P_{3 / 2}$ & $8.4898 \times 10^{-6}$ & - \\
$3 S_{1 / 2}$ & $2.4843 \times 10^{-7}$ & $2.3367 \times 10^{-6}$ \\
$3 P_{1 / 2}$ & $2.4843 \times 10^{-7}$ & - \\
$3 P_{3 / 2}$ & $7.4543 \times 10^{-7}$ & - \\
$3 D_{3 / 2}$ & $7.4543 \times 10^{-7}$ & - \\
$3 D_{5 / 2}$ & $1.7393 \times 10^{-6}$ & - \\
\hline
\end{tabular}

The nuclear size corrections to relativistic electron, is more effective on the lower $s$ energy levels and in heavy nuclei, as the effect varied directly with the nuclear size and inversely with the principal quantum number, $n$. A large number of publications (see, for example [21,34-38] showed that the nuclear finite-size effects, which is largest for $s$ levels, are more important for higher atomic nuclei and muonic atoms, since for muon the effects are enhanced by $\left(m_{\mathrm{e}} / m_{\mu}\right)^{2} \sim 10^{-5}$.

\section{Conclusion}

In this study a simple model satisfying the relationship between nuclear size correction to relativistic electron and electron energy state was developed to predict the energy level variation as a function of the size of the nucleus. This suggested application of analytical methods in solving relativistic equation that involved the finite-size nucleus through time independent perturbation theory. This study justifies the effects of nuclear size on relativistic electron and the measured values are of greatest interest since it will reveal significant changes of the nuclear structure and may also improve the knowledge of fine structure effect. The fine structure of hydrogen atom is important in atomic physics as it brings the idea of relativistic quantum mechanics and an important driving force in theoretical developments of atomic physics after it is experimentally discovered. 
Aliyu Adamu etal./ NIPES Journal of Science and Technology Research 2(3) 2020 pp. 36-44

\section{References}

[1] Maclay, G. J. (2019). History and Some Aspects of the Lamb Shift. Physics, Volume 2, Number 8, page $105-$ 149.

[2] Meka, F. (2020). Investigating Muonic Hydrogen Atom Energy Spectrum Using Perturbation Theory in Lowest Order. Advances in Physics Theories and Applications. Volume 83, page 7-21.

[3] Schrödinger, E. (1926).QuantisierungalsEigenwert-Problem.Annalen der Physik Volume 80, page 437.

[4] Landau, L. D. and Lifshitz, E. M., (1991). Quantum Mechanics, Non-relativistic Theory, Volume 3 of Course of Theoretical Physics. Third edition, Pergamon Press, Oxford, England. page 119.

[5] Greiner, W. (2001). Quantum Mechanics: An Introduction. Fourth Edition. Springer, Berlin, Germany, page 181, $220-227$.

[6] Herzberg, G. (1944). Atomic Spectra and Atomic Structure. Dover: Mineola, New York.

[7] White, H. E. (1934). Introduction to Atomic Spectra. (McGraw-Hill Book Company, New York), Chap. 8. New York, 1934.

[8] Sala, O., Araki, K. and Noda, L. K. (1999). A Procedure to Obtain the Effective Nuclear Charge from the Atomic Spectrum of Sodium. Journal of Chemical Education. Volume 76, Number 9, page 1269-1271.

[9] Adamu, A., Tartius, P.and Amshi, S. A., (2016). The Spectroscopy of Single Electron and Muonic Atoms. Journal of Physical Science and Innovations, Vol. 8, No. 2, pp $1-13$.

[10] Adamu A., (2016): Corrections to the Energy Levels of Finite - Size Nuclei due to Fluctuating Electromagnetic Fields in Vacuum. J-NAMP. Vol. 36, (July Issue), pp 215 - 222.

[11] Adamu, A. andNgadda, Y. H., (2015): The Nuclear Finite-Size Corrections to Energies of $n=1, n=2$ and $n=3$ States of Hydrogen Atom.J - NAMP. Vol. 30, pp $137-137$.

[12] Hernandez, O. J., Ji, C., Bacca, S., Dinur, N. N. andBarnea, N. (2014). Improved estimates of the nuclear structure corrections in $\mu$ D. arXiv:1406.5230v1 [nucl-th] 19 Jun 2014.

[13] Antognini, A., Kottmann, F., Biraben, F., Indelicato, P., Nez, F. and Pohl, R. (2013). Theory of the 2S-2P Lamb shift and 2S hyperfine splitting in muonic hydrogen. Annals of Physics. Volume 331, page 127-145.

[14] Bruch, R., Heilig, K., Kaletta, D., Steudel, A. and Wendlandt, D. (1969). Nuclear Volume and Mass Effect in the Optical Isotope Shift of Light Elements. Journal de Physique Colloques, Volume 30 (C1), page C1-51-C1-58.

[15] Mohammadi, S., Giv, B. N. and Shakib, N. S. (2017). Energy Levels Calculations of ${ }^{24} A l$ and ${ }^{25} A l$ Isotopes. Nuclear Science. Volume 2, Number 1, page 1-4.

[16] Mohr, P. J., Taylor, B. N. and Newel, D. B. (2012). CODATA recommended values of the fundamental physical constants: 2010. Reviews of Modern Physics. Volume 84, page 1527 - 1605.

[17] Galván, A. P., Zhao, Y. and Orozco, L. A. (2008). Measurement of the hyperfine splitting of the $6 S^{1 / 2}$ level in rubidium. Physical Review A78, 0125022008.

[18] Das, A. and Sidharth, B. G. (2015). Revisiting the Lamb Shift.Electronic Journal of Theoretical Physics. Volume 12, Number IYL15-34, page 139-152.

[19] Adamu, A., Hassan, M., Dikwa, M. K. and Amshi, S. A., (2018). Determination of Nuclear Structure Effects on Atomic Spectra by Applying Rayleigh-Schrödinger Perturbation Theory. American Journal of Quantum Chemistry and Molecular Spectroscopy. Volume 2, Number 2, page 39-51.

[20] Deck, R. T., Amar, J. G. and Fralick, G. (2005). Nuclear Size Corrections to the Energy Levels of Single-electron and -Muon Atoms. Journal of Physics B: Atomic Molecular and Optical Physics, Volume 38, page 2173 - 2186.

[21] Niri, B. N. and Anjami, A. (2018). Nuclear Size Corrections to the Energy Levels of Single-Electron Atoms. Nuclear Science. Volume 3, Number 1, page $1-8$.

[22] Hofstadter, R. and McAllister, R. W. (1955). Electron Scattering from the Proton. Physical Review. Volume 98, page 217.

[23] McAllister, R. W. and Hofstadter, R. (1956). Elastic Scattering of 188-Mev Electrons from the Proton and the Alpha Particle. Physical Review. Volume 102, page 851.

[24] Yearian, M. R. and Hofstadter, R. (1958). Magnetic Form Factor of Neutron. Physical Review. Volume 110, page 552.

[25] Ohmura, T. (1959). Effect of the Finite Size of the Proton on the Coulomb Energy of He ${ }^{3}$. Progress of Theoretical Physics. Volume 22 Issue 1, page 148 - 150.

[26] Adamu, A. and Ngadda, Y. H. (2017). Determination of Nuclear Potential Radii and its Parameter from Finite Size Nuclear Model.International Journal of Theoretical and Mathematical Physics. Volume 7, Number 1, page 9-13.

[27] Bayram, T., Akkoyun, S., Kara, S. O. and Sinan, A. (2013). New parameters for nuclear charge radius formulas.ACTA PHYSICA POLONICA B. Volume 44, number 8, page 1791 - 1799.

[28] Angeli, I. (2013). Manifestation of Non-Traditional Magic Nucleon Numbers in Nuclear Charge Radii. ACTA PHYSICA DEBRECINA. Volume 47, Number 7.

[29] Merino, C., Novikov, I. S. and ShabelskiY. M. (2009). Nuclear Radii Calculations in Various Theoretical Approaches for Nucleus-Nucleus Interactions. arXiv:0907.1697v1 [nucl-th] $10 \mathrm{Jul} 2009$. 
Aliyu Adamu etal./ NIPES Journal of Science and Technology Research

2(3) 2020 pp. 36-44

[30] Patoary, A. M. and Oreshkina, N. S. (2018). Finite nuclear size effect to the fine structure of heavy muonic atoms. The European Physical Journal D. Volume 72, page 54.

[31] Martensson-Pendrill, A. M. and Gustavsson, M. G. H. (2003). Handbook of Molecular Physics and Quantum Chemistry. John Wiley \& Sons, Ltd, Chichester, Volume 1, Part 6, Chapter 30, page 477 - 484. Edited by Stephen Wilson.

[32] Adamu, A. andNgadda, Y. H., (2014). The Effect of $1^{\text {st }}$ Order Time Independent Perturbation on the Finite Size of the Nuclei of Atoms.International Journal of Theoretical and Mathematical Physics. Volume 28, Number 1, page $333-339$.

[33] Adamu A. and Ngadda Y. H., (2017): Determination of Nuclear Potential Radii and Its Parameter from Finite Size Nuclear Model. International Journal of Theoretical and Mathematical Physics. Volume 7, Number 1, page 9-13.

[34] Borie, E. (2012). Lamb Shift in Light Muonic Atoms - Revisited. Annals of Physics. Volume 327, page 733763.

[35] Godunov, S .I. and M. I. Vysotsky, (2013). The Dependence of the Atomic Energy Levels on a Super Strong Magnetic Field with Account of a Finite Nucleus Radius and Mass, arXiv:1304.7940v1 [hep-ph].

[36] El Shabshiry, M., Ismaeel, S. M. E. and Abdel-Mageed, M. M. (2015). Finite Size Uehling Corrections in Energy Levels of Hydrogen and Muonic Hydrogen Atom. IOSR Journal of Applied Physics (IOSR-JAP), Volume 7, Issue 5 Ver. I, page $60-66$.

[37] Krane, K. S. (1988). Introductory Nuclear Physics. John Wiley and Sons Inc., New York. Page 49.

[38] Neznamov, V. P. and Safronov, I. I. (2015). A new Method for Solving the Z > 137 Problem and for Determination of Energy Levels of Hydrogen-Like Atoms. arXiv: 1307.0209 V3. 
Aliyu Adamu etal./ NIPES Journal of Science and Technology Research 2(3) 2020 pp. 36-44

\section{Appendix}

The predicted $\log \left[\Delta E_{r}(n, j)\right]$ for $n=1,2, \ldots, 100$ states of hydrogen

\begin{tabular}{cc}
\hline $\boldsymbol{n}$ & $\mathbf{L o g} \Delta \boldsymbol{E}_{\boldsymbol{r}}(\boldsymbol{n}, \boldsymbol{j})$ \\
\hline 001 & 3.2310 \\
002 & 1.4288 \\
003 & 0.3746 \\
004 & -0.3734 \\
005 & -0.9535 \\
006 & -1.4276 \\
007 & -1.8284 \\
008 & -2.1756 \\
009 & -2.4818 \\
010 & -2.7557 \\
011 & -3.0035 \\
012 & -3.2298 \\
013 & -3.4379 \\
014 & -3.6306 \\
015 & -3.8099 \\
016 & -3.9777 \\
017 & -4.1354 \\
018 & -4.2840 \\
019 & -4.4245 \\
020 & -4.5579 \\
021 & -4.6848 \\
022 & -4.8057 \\
023 & -4.9213 \\
024 & -5.0319 \\
025 & -5.1381 \\
026 & -5.2401 \\
027 & -5.3382 \\
028 & -5.4327 \\
029 & -5.5240 \\
030 & -5.6121 \\
031 & -5.6974 \\
032 & -5.7799 \\
033 & -5.8599 \\
\hline &
\end{tabular}

\begin{tabular}{ll}
\hline 034 & -5.9375 \\
035 & -6.0129 \\
036 & -6.0862 \\
037 & -6.1574 \\
038 & -6.2267 \\
039 & -6.2943 \\
040 & -6.3601 \\
041 & -6.4243 \\
042 & -6.4869 \\
043 & -6.5481 \\
044 & -6.6079 \\
045 & -6.6663 \\
046 & -6.7235 \\
047 & -6.7794 \\
048 & -6.8341 \\
049 & -6.8877 \\
050 & -6.9403 \\
051 & -6.9918 \\
052 & -7.0422 \\
053 & -7.0918 \\
054 & -7.1404 \\
055 & -7.1881 \\
056 & -7.2349 \\
057 & -7.2809 \\
058 & -7.3262 \\
059 & -7.3706 \\
060 & -7.4143 \\
061 & -7.4573 \\
062 & -7.4996 \\
063 & -7.5412 \\
064 & -7.5821 \\
065 & -7.6224 \\
066 & -7.6621 \\
067 & -7.7012 \\
\hline &
\end{tabular}

\begin{tabular}{|c|c|}
\hline 068 & -7.7397 \\
\hline 069 & -7.7777 \\
\hline 070 & -7.8151 \\
\hline 071 & -7.8520 \\
\hline 072 & -7.8883 \\
\hline 073 & -7.9242 \\
\hline 074 & -7.9596 \\
\hline 075 & -7.9945 \\
\hline 076 & -8.0289 \\
\hline 077 & -8.0629 \\
\hline 078 & -8.0964 \\
\hline 079 & -8.1296 \\
\hline 080 & -8.1623 \\
\hline 081 & -8.1946 \\
\hline 082 & -8.2265 \\
\hline 083 & -8.2580 \\
\hline 084 & -8.2891 \\
\hline 085 & -8.3199 \\
\hline 086 & -8.3503 \\
\hline 087 & -8.3804 \\
\hline 088 & -8.4101 \\
\hline 089 & -8.4395 \\
\hline 090 & -8.4685 \\
\hline 091 & -8.4972 \\
\hline 092 & -8.5257 \\
\hline 093 & -8.5538 \\
\hline 094 & -8.5816 \\
\hline 095 & -8.6091 \\
\hline 096 & -8.6363 \\
\hline 097 & -8.6633 \\
\hline 098 & -8.6899 \\
\hline 099 & -8.7163 \\
\hline 100 & -8.7424 \\
\hline
\end{tabular}

